\title{
Unattended Autonomous Mission and System Management of an Unmanned Seaplane
}

\author{
Ryan D. Eubank* and Ella M. Atkins ${ }^{\dagger}$ \\ University of Michigan, Ann Arbor, MI, 48109, USA
}

The Flying Fish autonomous solar-energy-harvesting seaplane was designed for persistent deployment on the open ocean as a combination aerial-observation and drifting-buoy platform. Two generations of field-tested vehicles have demonstrated continuous sequences of self-initiated autonomous flight operations in marine and freshwater environments. The addition of solar energy collection in the second-generation vehicle brings extended-toperpetual system deployment within reach. This paper presents the implementation and preliminary results of the modeling and planning utilities meant to achieve energy-aware mission management for safe, long-term unattended, vehicle deployment. Results are presented from flight-test-derived simulations and models.

\section{Nomenclature}

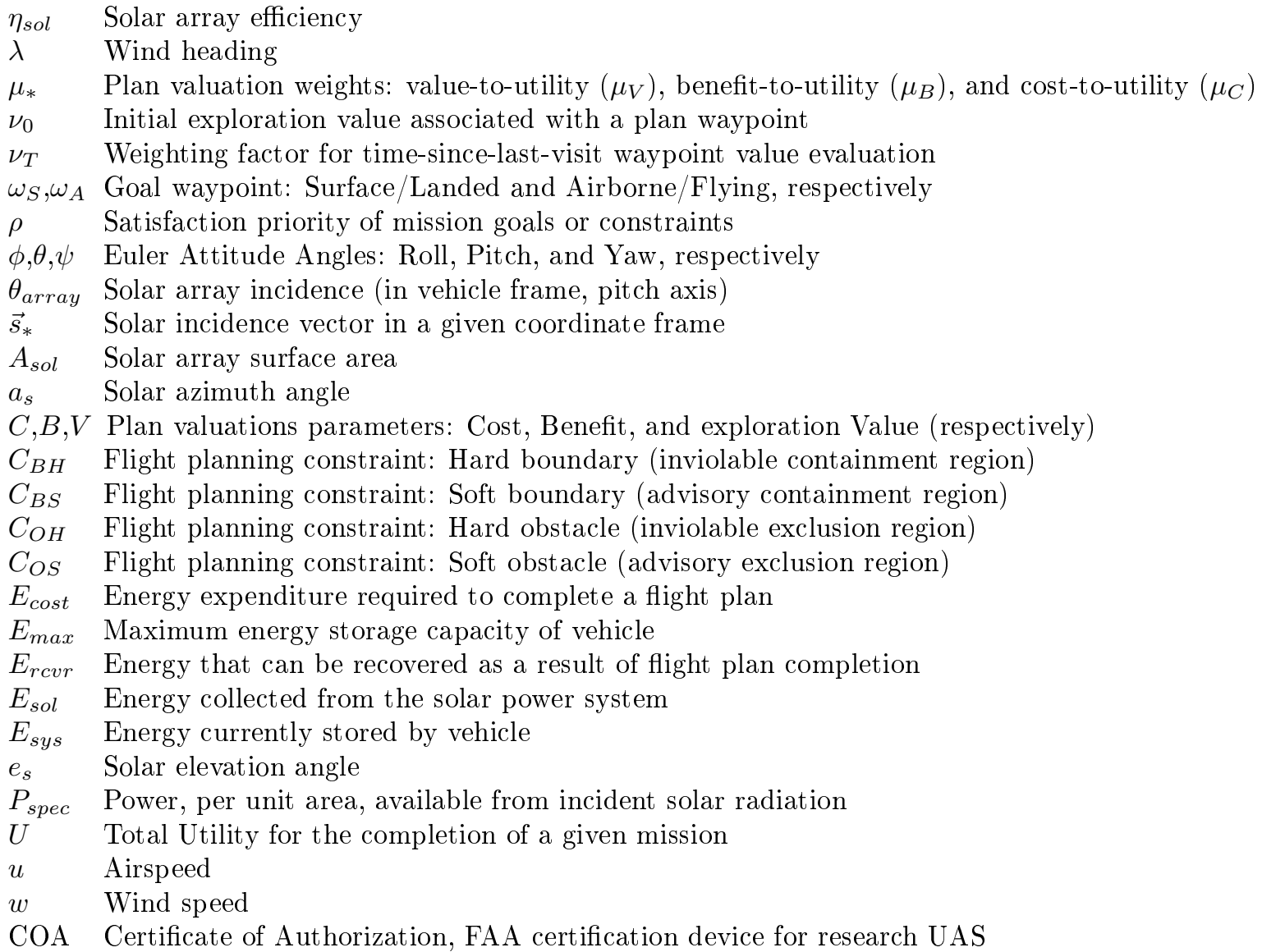

${ }^{*}$ Graduate Research Assistant, Aerospace Engineering, 1320 Beal Ave - 2016 FXB, Student Member

${ }^{\dagger}$ Associate Professor, Aerospace Engineering, 1320 Beal Ave - 3009 FXB, Associate Fellow 
FMS Flight Management System

NREL National Renewable Energy Laboratory

UAS Unmanned Aerial System

\section{Introduction}

T

HE Flying Fish system represents a new unmanned aerial system (UAS) operating paradigm wherein the onboard avionics system must manage not just a single flight but a potentially-perpetual series of flight and surface maneuvers for continuous operator-independent deployment. The first element of this process, the execution of sequential self-managed flight operations, has been demonstrated over the course of two vehicle programs ${ }^{1,2}$ wherein a multi-mode gain-scheduled PD control scheme has proven effective for directing stable flight operations from auto-takeoff through auto-landing. ${ }^{3}$

To move beyond basic flight operations the onboard flight management system (FMS) must plan the pace and order of goal satisfaction, monitor vehicle systems, and diagnose failures to ensure that the system remains survivable, long-term, without recovery. The FMS planning utility must be able to forecast energy collection and expenditure to plan sequential flights to achieve primary, auxiliary, and opportunistic mission goals. The planner must also account for overnight survivability and have reasonable estimates of, and responses to, the effects of inclement weather on solar energy and vehicle position. The system must be capable of planning obstacle avoidance maneuvers given a priori obstacle data and re-plan missions if unexpected obstacles are encountered. The collected system requirements dictate a need for high level data interpretation and decision mechanisms that can still be executed with the limited resources of an embedded computer.

The remainder of this introductory section briefly presents background information on the existing Flying Fish FMS. Section II details the models utilized by the estimation and planning system that is subsequently presented in section III. Section IV features simulated deployment results and the results of basic mission planning strategies. The paper concludes in section $\mathrm{V}$ with a discussion of the capabilities and limitations of the current system and the ongoing efforts to add fidelity and functionality to the Flying Fish FMS.

\section{I.A. Background}

Flight management systems first became standard equipment on major commercial aircraft in 1982 where they were used to offload vehicle management tasks from the pilot and, as a result of spiking aviation fuel costs in the preceding decade, to plan and guide fuel-optimized trajectories. ${ }^{4}$ The proliferation of these systems marked a major turning point for flight software as the FMS was, up to that point, the most software intensive system onboard a commercial aircraft. The purpose of the FMS goes beyond that of an autopilot to include: flight planning, navigation, guidance, performance prediction and optimization, communication, control, and the management of user interfaces. The FMS is now part of the standard equipment of all commercial aircraft and in the age of the glass cockpit and rising fuel costs it plays an even greater roll in system management and flight optimization. Flight management

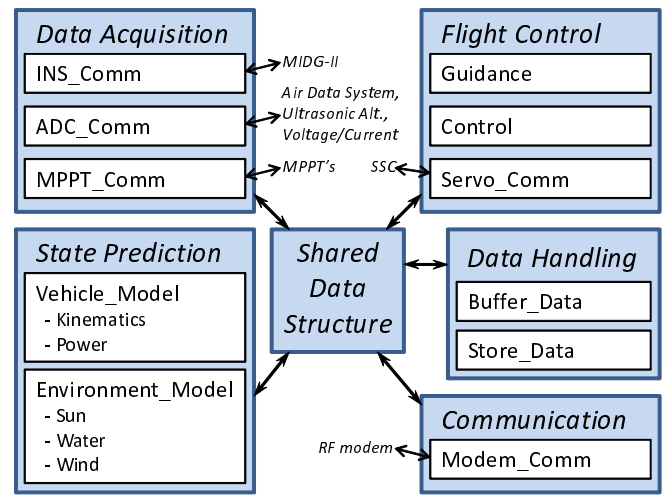

Figure 1: Wolverine FMS Architecture systems are generally comprised of some sort of flight management computer executing the collected FMS software, some communication equipment, and a user control/display unit that serves as an interface for the FMS user.

The first-generation Flying Fish FMS managed: vehicle sensors, communications, data storage, navigation, guidance, and flight control and in the second-generation began to add fault detection routines ${ }^{5}$ and performance estimation. The Flying Fish FMS is based on the Wolverine FMS, an open-source flight management software package developed in the Autonomous Aerospace Systems Laboratory (A2Sys) at the University of Michigan. ${ }^{6}$ The Wolverine FMS has been adapted to four other A2Sys-affiliated platforms to date, including an aerobatic UAS, ${ }^{7}$ a highly-flexible flying-wing $\mathrm{UAS}^{8}{ }^{8}$ and the first $^{3}$ and second-generation Flying Fish UAS. ${ }^{9}$ Figure 1 shows the structure of the Wolverine FMS architecture as it has been adapted 
to Flying Fish. The prediction portion of the Wolverine FMS executes two procedures (Vehicle_Model and Environment_Model) highlighting the two broad categories of prediction required for energy-aware flight management and planning. The Vehicle_Model procedure (Fig. 1), divided between motion and energy modules, provides the mechanisms required for energy state estimation and flight execution prediction. The Environment_Model procedure (Fig. 1), divided into solar, wind, and water modules, provides estimates of short and long term environmental conditions for planning purposes. Details of the preliminary models, the flight management computer, avionics, and customized Wolverine-FMS implementation can be found in Ref. 9.

\section{System and Environmental Models}

The Flying Fish FMS relies on a number of modeling faculties for the computation of performance estimates and mission plans. These models generally fall into two broad categories which, as first indicated in the previous section, are classified into: 1) vehicle models, and 2) environment models. Vehicle models provide the FMS with tools for estimating vehicle performance and sub-system states/processes while environment models provide estimates of the external states and processes that effect the UAS mission. The basic models employed for the Flying Fish FMS were proposed in a previous publication. ${ }^{9}$ For clarity this section provides a high-level overview of the existing models together with the new/updated models but will omit any previously presented derivation, implementation, and background information.

\section{II.A. Vehicle Models}

Primary motion estimation and vehicle trajectory propagation utilize a wind-aware bank-to-turn kinematic model (Eqns. 1-3). Based on the well-studied unicycle model with bank-to-turn augmented turn dynamics ${ }^{10}$ the Flying Fish model adds wind to the translational dynamics as wind effects are a critical consideration for seaplane deployment. Here $g$ is gravitational acceleration, $\phi$ and $\psi$ are the bank and heading angles, respectively, and $x$ and $y$ are the Cartesian coordinates of the aircraft in the navigation-axes (locally-level inertial frame with the $x$-axis pointed North and $y$-axis East). The model is subject to the local wind speed $w$, wind heading $\lambda$, and the airspeed $u$ of the aircraft.

$$
\begin{gathered}
\dot{x}=u \cos (\psi)+w \cos (\lambda) \\
\dot{y}=u \sin (\psi)+w \sin (\lambda) \\
\dot{\psi}=\frac{g \tan (\phi)}{u}
\end{gathered}
$$

To complete the vehicle motion model we make several assumptions about seaplane motion, add parameters from curve-fit flight data, and utilize a length-optimal path generation method to connect waypoints. The primary assumption governing our motion model is that takeoff and landing can, and will, be flown into the wind. This assumption is both reasonable and practical as non-slipping/non-skidding flight is aerodynamically efficient and naturally adopted by a seaplane in unconstrained transit over water or, when airborne, during stabilized stick-free flight. The takeoff, climb, and decent performance of the vehicle are modeled on extensive flight data that yielded consistent accelerations, velocity thresholds, and average durations for each of those mission segments. Finally, classical dubins paths are employed for waypoint-to-waypoint trajectory generation. ${ }^{11}$ Dubins paths are provably minimum-length paths composed of arcs (flown at a fixed - typically maximum - turn-rate) connected by straight-line cruise segments. While flight vehicles cannot fly the exact course of a dubins path due to the implicit requirement for instantaneous acceleration changes, these paths serve as an useful, smooth, piece-wise continuous, minimum-length baseline path that can still be flown with reasonable accuracy given good control and guidance strategies.

In addition to the motion model an energy-aware FMS must maintain accurate models of power subsystems including solar-energy-collection performance, battery performance, and propulsion and avionics loads. The energy collected by a solar array can be modeled by he integral of the spectral power density $\left(\mathrm{W} / \mathrm{m}^{2}\right)$ of the incident sunlight with the cosine of the solar incidence angle and the area $\left(A_{\text {sol }}\right)$ and efficiency $\left(\eta_{\text {sol }}\right)$ of the solar array :

$$
E_{\text {sol }}=\int_{t_{0}}^{t_{f}} \eta_{\text {sol }} A_{\text {sol }} P_{\text {spec }}(t) \cos \left(\angle \vec{s}_{A}(t)\right) d t
$$


The solar incidence vector $\left(\vec{s}_{A}\right)$, in the coordinate frame of the solar array, is determined from the inertialframe solar incidence vector $\left(\vec{s}_{I}\right)$ via a sequence of rotations about the navigation axes (locally-level, x-axis North, y-axis East) by the Euler-Angles of the vehicle $\{\phi, \theta, \psi\}$ and the vehicle-relative solar-array pitch angle $\left(\theta_{\text {array }}\right)$ :

$$
\vec{s}_{A}=R_{x}\left(\theta+\theta_{\text {array }}\right) R_{z}(\psi) R_{x}(\pi) \vec{s}_{I}
$$

The cosine of the solar incidence to the array can subsequently be determined by a dot-product of the array-frame incidence vector, $\vec{s}_{A}$, and the vertical array-normal vector (namely the z-axis of the array-frame coordinate system). The solar spectral power density $P_{\text {spec }}(t)$ and the inertial-frame solar incidence vector $\vec{s}_{I}(t)$ are environment processes and are discussed below.

The authors previously proposed a battery model based on curve-fits of charge/discharge curves of laboratory and flight test data from the actual Flying Fish lithium-polymer flight batteries. ${ }^{9}$ While the linear-fit was true to measured data the model was unable to represent the dynamic response of lithium batteries to heavy loading. A number of alternative lithium battery models have been developed in recent years ${ }^{13,14}$ with much of the research driven by growing interest in electric transportation. ${ }^{12,15}$ The newest Flying Fish battery model is adapted from a model presented in a National Renewable Energy Laboratory (NREL) lithium-battery technical report. ${ }^{12}$

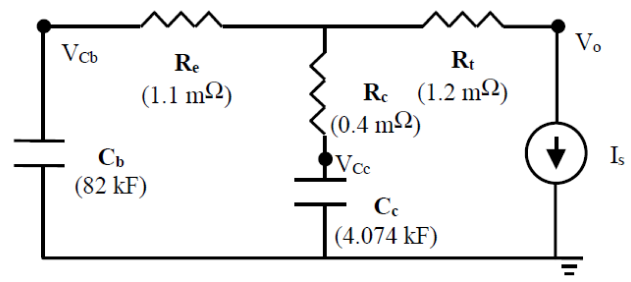

Figure 2: NREL-Saft Battery Model ${ }^{12}$

The model was attributed to lithium-battery manufacturer Saft, but seems to be a variation on previously know models. ${ }^{13,14}$ The NREL-Saft model represents the battery as a parallel resister-capacitor network with input/output impedance (Fig. 2). The linear model of this system is given by:

$$
\begin{gathered}
{\left[\begin{array}{c}
\dot{V}_{C b} \\
\dot{V}_{C c}
\end{array}\right]=\left[\begin{array}{cc}
\frac{-1}{C_{b}\left(R_{e}+R_{c}\right)} & \frac{1}{C_{b}\left(R_{e}+R_{c}\right)} \\
\frac{1}{C_{c}\left(R_{e}+R_{c}\right)} & \frac{-1}{C_{c}\left(R_{e}+R_{c}\right)}
\end{array}\right]\left[\begin{array}{c}
V_{C b} \\
V_{C c}
\end{array}\right]+\left[\begin{array}{c}
\frac{-R_{c}}{C_{b}\left(R_{e}+R_{c}\right)} \\
\frac{-1}{C_{C}}+\frac{R_{c}}{C_{c}\left(R_{e}+R_{c}\right)}
\end{array}\right]\left[I_{s}\right]} \\
{\left[V_{o}\right]=\left[\begin{array}{ll}
\frac{R_{C}}{\left(R_{e}+R_{c}\right)} & \frac{R_{e}}{\left(R_{e}+R_{c}\right)}
\end{array}\right]\left[\begin{array}{c}
V_{C b} \\
V_{C c}
\end{array}\right]-\left[R_{t}+\frac{R_{c} R_{e}}{\left(R_{e}+R_{c}\right)}\right]\left[I_{s}\right]}
\end{gathered}
$$

The model treats battery energy as charge on a large capacitor, with the charge/discharge response set by the output impedance, and a smaller capacitor that models the nonlinear potential-reduction region of a lithium battery under heavy load. The model has been tuned to the capacity and voltage of a single Flying Fish battery cell by the appropriate selection of impedance and capacitance $\left(R_{e}=1.1 \mathrm{~m} \Omega, R_{c}=0.4 \mathrm{~m} \Omega, R_{t}=2.2 \mathrm{~m} \Omega, C_{b}=\right.$ $\left.18.45 \mathrm{kF}, C_{c}=4.0 \mathrm{kF}\right)$. The authors have also changed the sign of the current input $I_{s}$ block of Eq. (7) which is a suspected error in the original NREL report as the published model gives increasing voltage under heavy loads. Series battery voltage is recovered by scaling the model to the cell-count of a Flying Fish battery pack and battery-bank capacity is handled by dividing loads over the number of batteries in each bank. The Flying Fish has two large

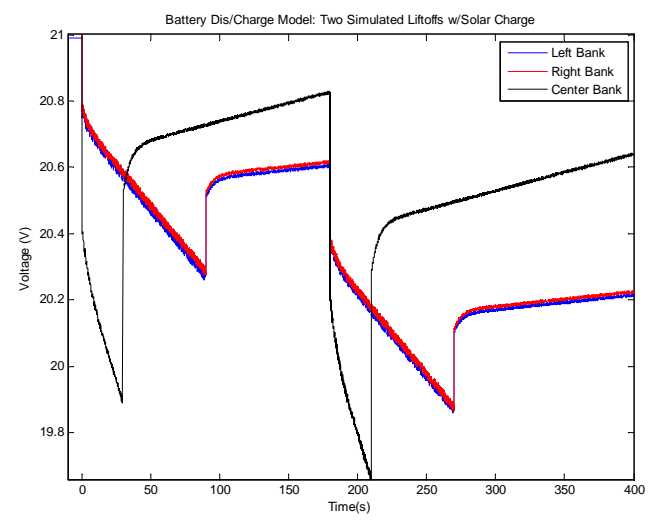

Figure 3: Battery Simulation: Two Flights main battery banks charged by the solar array (loaded by the avionics and primary propulsion) and a smaller central bank that is charged by the primary banks (and loaded by the avionics and an auxiliary propulsion system). A simulation of the Flying Fish power system with the NREL-Saft battery model over two 1.5-minute flight cycles with solar charging is shown in Fig. 3.

Propulsion loads are modeled primarily from flight-derived parameters. A calibration curve from throttle position to motor current has been developed for both the main and boost propulsion systems. This curve can be applied to the throttle vector over a flight profile to determine system loads and then, by applying the battery model, to compute cumulative energy expenditures. Throttle settings for each stage of flight: takeoff, climb, cruise, and descent have been extracted from flight data and are used to estimate the power required 
for each leg of a flight. Once flight times are computed for each leg of a mission the total energy expenditure can be determined. This process is straightforward for linear flight segments but an averaging/integration mechanism is required to propagate power and energy consumption over curved trajectories. Currently the model assumes that the straight-line cruise segment of each dubins turn-fly-turn trajectory serves as a reasonable average flight direction for that segment. Subsequently a solution of the the wind-heading velocity triangle is used to determine the slipping-flight speed along the cruise path which divides the entire turn-fly-turn segment length to produce segment flight time. The final assumption used for mission segment modeling is that the difference in dubins trajectory length between turning at a waypoint and turning before a waypoint, to intercept it on an arc, are negligible.

All avionics loads are assumed, with the exception of surface-deployed payloads, to be active during flight but the system is subject to a number of loads that are "sheddable" when the vehicles is on the water. Assuming that the vehicle must maintain situational awareness on the water the always-on fixed "hotel" loads include: avionics computer (ACPU), inertial navigation system (INS), wireless communications (can be idled, but must monitor command channels), and the miscellaneous regulator/interface overhead. The routinely sheddable loads on the water include the control actuation mechanisms (regulators, servos, \& controllers), and the ultra-sonic altimeter. Auxiliary payloads are assumed to be "any time" sheddable for the purposes of survival. Table 1 provides an estimate of best case fixed and sheddable loads onboard a seaplane UAS in the same class as the Flying Fish. To be clear, this analysis ignores the complexity that load-shedding

Table 1: Flying Fish Fixed and Sheddable Loads

(a) Fixed Hotel Loads

(b) Sheddable Loads

\begin{tabular}{|c|c|c|c|c|c|c|c|}
\hline & $P_{\text {on }}(\mathrm{W})$ & $P_{\text {idle }}(\mathrm{W})$ & $P_{\text {sleep }}(\mathrm{W})$ & & $P_{\text {on }}(\mathrm{W})$ & $P_{\text {idle }}(\mathrm{W})$ & $P_{\text {off }}(\mathrm{W})$ \\
\hline \hline ACPU & 1.0 & - & - & Ultrasonic & 1.47 & - & 0.0 \\
\hline Modem & 4.83 & 0.7 & 0.133 & Servo Controller & 0.5 & - & 0.0 \\
\hline INS & 1.2 & - & - & Control Servos (ea) & 3.15 & 0.264 & 0.0 \\
\hline Misc & 0.9 & - & - & Servo Receiver & 0.075 & - & 0.0 \\
\hline
\end{tabular}

imparts to vehicle design. Whenever a load is made to be sheddable additional power switching must be added which increases the complexity of both the wiring and control systems and creates additional possible failure points. There are also safety issues to be considered if a critical load might be accidentally "shed" during flight. For example, while the Flying Fish has the infrastructure to switch the the servo mechanisms, they have remained un-switched throughout development for the sake of safety and reliability. Operating in this fashion the best-case Flying Fish load-shedding can only assume idle servos and ultrasonic-altimeter deactivation.

\section{II.B. Environment Models}

The Flying Fish solar power model is based on NREL-published solar position and irradiation calculators. ${ }^{16,17}$ These models are capable of solving for the sun azimuth $\left(a_{s}\right)$ and elevation $\left(e_{s}\right)$ angles and atmosphere-corrected solar power density $\left(P_{\text {spec }}(t)\right)$ for a given date and geographic location. The inertial-frame solar incidence vector can subsequently be constructed from the sun azimuth and elevation angles using Eq. 8. With these environmental processes modeled we can apply the vehicle solarpower model in Eq. (4) to determine the available power (integrand) and energy (integral). An example of a daily solar energy simulation showing the solar incidence angle (on a $1.34 \mathrm{~m}^{2}$ horizontal solar array), total atmospheric incident solar power, and efficiency-scaled harvestable solar power is presented in

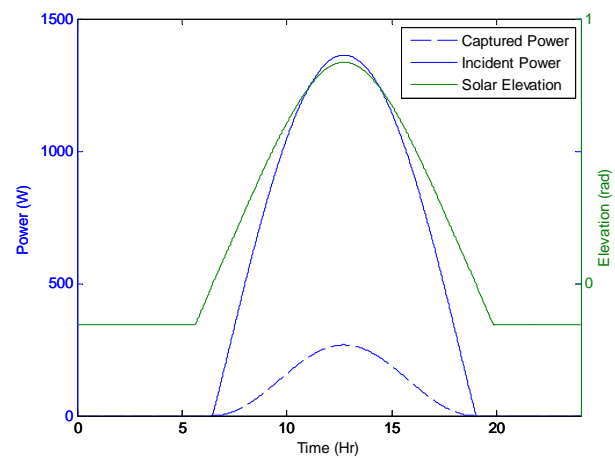

Figure 4: Daily Solar Energy Model 
Fig. 4.

$$
\vec{s}_{I}=\left[\begin{array}{c}
\cos \left(a_{s}\right) \cos \left(e_{s}\right) \\
\sin \left(a_{s}\right) \cos \left(e_{s}\right) \\
\sin \left(e_{s}\right)
\end{array}\right]
$$

Several additional environment phenomena, including wind and ocean currents, are modeled as random nonzero-mean noise processes. The wind process directly effects both the vehicle drift rate/direction and vehicle flight performance. Ocean currents directly effect the vehicle drift rate and direction. Because it is difficult, and not strictly necessary, to separate the contributions of ocean currents from wind in the cumulative drift behavior the drift model treats drift as a single process rather than a combination of a windbased and current-based process. The characteristics of these signals are drawn from vehicle measurements on each deployment and in the simulation and planning systems they generally simplify to average values with error-bounds.

\section{Mission Planner}

In order for the deployed Flying Fish to negotiate a series of potential flight goals it must have both a defined mission domain and an optimization mechanism to evaluate and trade mission value against cost and long-term energy balance. To this end the system requires a robust planning capability that is able to assemble and revise daily operational plans to maintain a balanced energy budget and provide for overnight survivability. For this purpose we first introduce a uniform planner entry format that is used to represent both missions and constraints. We subsequently describe the types and implementation of constraints followed by a description of the environment and missions in the planning domain. For missions we pay particular attention to the special considerations and assumptions associated with planning for an energy-harvesting seaplane. We then describe the mechanisms for constructing and evaluating mission plans. An illustrative example from the planner is presented below and full daily mission plan is presented in the results section IV.

Within the Flying Fish planner every entry, be it a goal waypoint or constraint, takes the form of an augmented waypoint that is endowed with a uniform set of attributes: time, position, velocity, Euler-angle attitude, spacial dimensions, activation value, execution priority, description, and type. Not all entries use all of these attributes but almost any type of entry can be represented without additional attributes which makes bookkeeping and software development more straightforward. Every planner entry takes the form:

$$
\begin{aligned}
P_{i} & =\{\text { type, time, position, velocity, attitude, dimensions, priority, value, description }\} \\
& =\left\{\operatorname{TYP}, t,(x, y, z),(\dot{x}, \dot{y}, \dot{z}),(\phi, \theta, \psi),\left(r_{1}, r_{2}, h\right), \rho, \nu_{o}, \mathrm{DES}\right\}
\end{aligned}
$$

We define four types of constraints: hard obstacles $\left(C_{O H}\right)$, soft obstacles $\left(C_{O S}\right)$, hard boundaries $\left(C_{B H}\right)$, and soft boundaries $\left(C_{B S}\right)$ and two types of goal waypoints: surface goals $\left(\omega_{S}\right)$, and aerial goals $\left(\omega_{A}\right)$. Constraint boundaries define containment regions for vehicle operations while constraint obstacles define exclusion regions. Hard and soft constraints are considered either inviolable to ensure vehicle survival or advisory, permitting violations for vehicle survival, respectively. Each goal's value $(\nu)$ is computed over time as the sum of the initial value attribute $\left(\nu_{o}\right)$ plus a weighted time-since-lastvisit incentive that encourages re-exploration: $\left(\nu_{T}\right)$ :

$$
\nu_{i}=\nu_{0, i}+\nu_{T, i} \cdot \Delta t
$$

Goal values are zeroed at the moment of goal satisfaction and subsequent value is derived only from

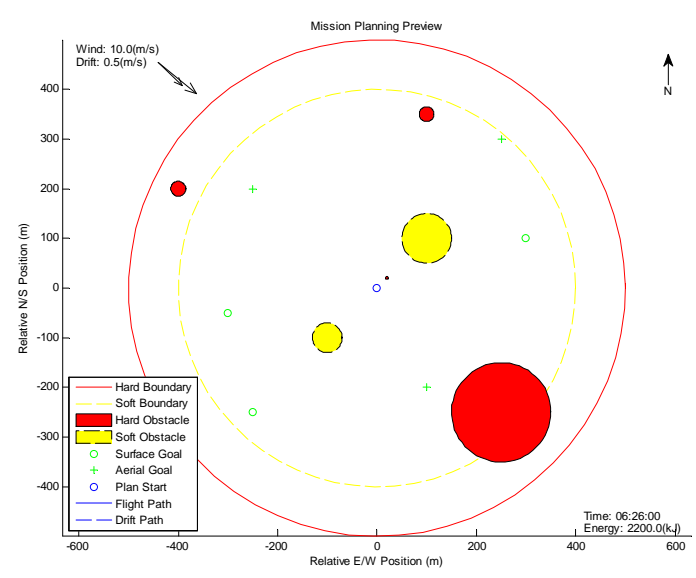

Figure 5: Planning Environment

the time-since-last-visit incentive. Priority is a separately considered waypoint/constraint valuation method. In the event that differentiation needs to be made between soft constraints or when goal values are insufficient to discriminate between goals the priority provides an auxiliary ranking. For example, given two 
soft constraints if one constraints must be violated for vehicle survival a secondary value may be required to deconflict the selection. Similarly, if initial goal waypoint values and time-since-last-visit valuations are insufficient to encode sufficient value to a critical goal, then a goal priority may be applied. Priorities are assigned values between 0 and 10, inclusive, with higher values indicating higher priority.

The environment of Flying Fish, and of the mission planner, is defined by the set of all known constraints and the environmental processes for which models were previously discussed. The environmental constraints are defined using the planner entry format with position, velocity, and size attributes and may include soft and hard boundaries and obstacles. An example constraint environment (with unused planner attributes omitted for brevity) is presented in tabular form in Table:2 and graphically in Fig. 5.

Table 2: Planner Environment Description

\begin{tabular}{|l|c|c|c|c|c|}
\hline & Type & Position & Velocity & Dimension & Description \\
\hline \hline$C_{B H, 1}$ & Hard Boundary & $(0,0,0)$ & $(0,0,0)$ & $(500,500,100)$ & "Shore Line" \\
\hline$C_{B S, 1}$ & Soft Boundary & $(0,0,0)$ & $(0,0,0)$ & $(400,400,500)$ & "Advised Airspace" \\
\hline$C_{O H, 1}$ & Hard Obstacle & $(-100,200,0)$ & $(-10,20,0)$ & $(15,15,15)$ & "Boat" \\
\hline$C_{O H, 2}$ & Hard Obstacle & $(20,20,0)$ & $(0,0,0)$ & $(3,3,4)$ & "Buoy" \\
\hline$C_{O H, 3}$ & Hard Obstacle & $(250,-300,0)$ & $(0,0,0)$ & $(150,150,5)$ & "Reef" \\
\hline$C_{O S, 1}$ & Soft Obstacle & $(100,-200,0)$ & $(1,-1,0)$ & $(50,50,0)$ & "Oil Slick" \\
\hline$C_{O S, 2}$ & Soft Obstacle & $(-100,-100,0)$ & $(0,-1,0)$ & $(30,30,0)$ & "Algae Bloom" \\
\hline
\end{tabular}

Flying Fish missions are specified in much the same way as any UAS except that there are a number of vehicular and environmental considerations that uniquely impact the missions and operations of an energyharvesting seaplane. Perhaps the largest difference, as previously discussed, is that Flying Fish cannot be assumed, a priori, to have a terminal mission plan; Flying Fish must continue to create, select, and fly goal-satisfying missions indefinitely to survive. The second major consideration for a seaplane UAS is that while operation over the ocean provides several clear operational benefits it also imparts several added challenges for mission planning. If surface obstacles are momentarily ignored it can be argued that the ocean provides during a given flight profile: 1) an effectively infinite landing surface, with 2) little to no air traffic, and 3) easily avoided surface traffic. Unfortunately the near infinite surface of the ocean is: 1) continuously changing shape, 2) subject to harsh environmental conditions, and 3) provides no guaranteed stationary loiter point on the water. From this last condition alone we find that significant mission activity may arise just from the specification of a hard or soft boundary. Environmental disturbances will naturally result in the traversal of most any fixed region on the water and subsequently require routine flight to avoid constraint violation. If solar-energy collection is also considered, the boundary-constraint mission results in the selection of surface goals that maximize the drift time across the constraint region in order to maximize energy collection. In general, a solar-power seaplane UAS planner must maximize energy recovery which will most often be accomplished by maximizing time spent in the lowest energy state (drift). Finally the planner must maintain awareness of system energy levels and respect the limits of the system capacity; no more energy can be harvested than the batteries can store nor can more energy be expended than the system can supply. As a result we ultimately define the Flying Fish mission as the satisfaction of zero or more surface goals $\omega_{S, i}$ and zero or more airborne goals $\omega_{A, i}$ subject to the set of all vehicle and environmental constraints. An example set of goals are given in Table 3 and are also graphically represented in Fig. 5 .

Table 3: Planner Mission Description

\begin{tabular}{|c|c|c|c|c|c|c|c|c|}
\hline & Type & Position & Vel. & Att. & Dim. & Priority & Value & Description \\
\hline \hline$w_{S, 1}$ & Surface & $(-250,-250,0)$ & - & - & - & 8 & 0 & "Point of Interest" \\
\hline$w_{S, 2}$ & Surface & $(-300,-50,0)$ & - & - & - & 7 & 0 & "Point of Interest" \\
\hline$w_{S, 3}$ & Surface & $(300,100,0)$ & - & - & - & 8 & 10 & "Point of Interest" \\
\hline$w_{A, 1}$ & Airborne & $(250,300,20)$ & - & - & - & 8 & 0 & "Point of Interest" \\
\hline$w_{A, 2}$ & Airborne & $(-250,200,20)$ & - & - & - & 8 & 0 & "Point of Interest" \\
\hline$w_{A, 3}$ & Airborne & $(100,-200,20)$ & - & - & - & 8 & 0 & "Point of Interest" \\
\hline
\end{tabular}




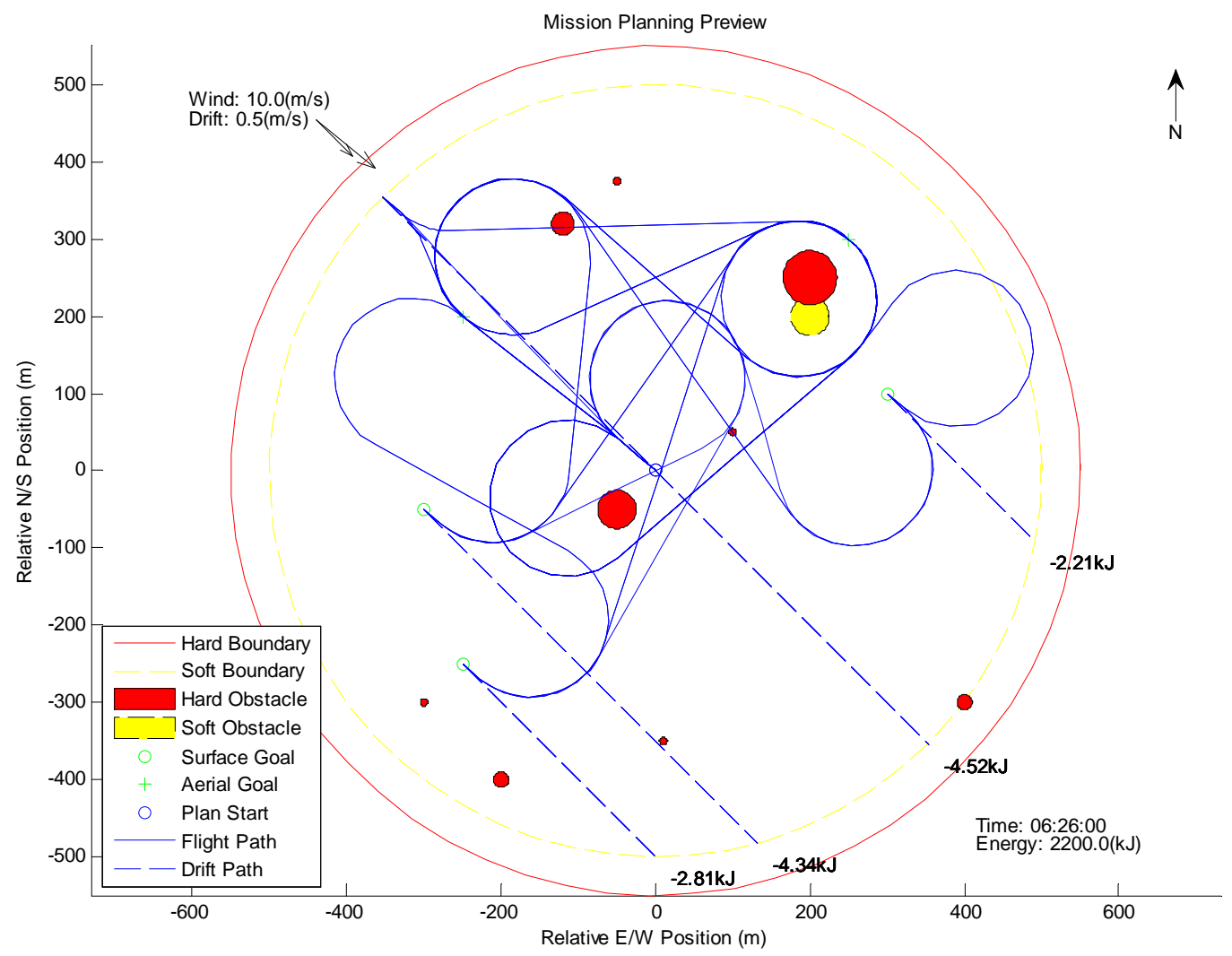

Figure 6: Expansion of All Possible Mission Trajectories

Given a constrained environment, mission description, and estimation models a planner can be enacted on the set of all possible mission plans to determine the best set of actions to take over the course of a single flight operation and ultimately over the course of a daily mission plan. A number of steps must be performed at each planning stage. First the planner assembles the combinatorial set of all waypoint sequences starting from the current drift location (including waypoints generated for constraint avoidance) and ending at a surface goal. Any sequence of waypoints that includes multiple surface goals or that has surface goals in the middle of a sequence are eliminated because each landing marks a new planning stage. For each remaining waypoint sequence the models discussed in section II are applied to develop flight trajectories satisfying each set of waypoints (Fig. 6). The models subsequently yield flight time $\left(T_{k}\right)$ and energy expended $\left(E_{\text {cost }, k}\right)$ over each path. The planner then uses the models to estimate the amount of energy that can be recovered $\left(E_{r c v r, k}\right)$ on a subsequent drift from each terminal waypoint. The planner assumes that the recovered energy is the lesser of either the energy available from drifting to the boundary $\left(E_{r c v r, k}\right)$ or the total energy that the vehicle can store $\left(E_{\max }-E_{\text {current }}\right)$. Given the energy cost and recovery associated with each path the planner computes the utility $\left(U_{k}\right)$ of each mission, given the a set of weights $\left\{\mu_{V}, \mu_{B}, \mu_{C}\right\}$ and the mission waypoint values $\left(\nu_{i}\right)$ :

$$
\begin{gathered}
V_{k}=\Sigma_{i}\left(\nu_{i}\right) \\
B_{k}=\min \left(E_{r c v r, k},\left(E_{\text {max }}-E_{\text {sys }}\right)\right) \\
C_{k}=E_{\text {cost }, k} \\
U_{k}=\mu_{B} \cdot B_{k}+\mu_{V} \cdot V_{k}+\mu_{C} \cdot C_{k}
\end{gathered}
$$

At every stage of planning a "no operation" (no-op) action exists wherein the planner may elect to drift until a boundary is encountered (or the system energy becomes full). The utility of the no-op mission (Eq. 14) assumes zero cost $\left(C_{n o-o p}=0\right)$ and zero value $\left(V_{n o-o p}=0\right)$ but has an energy-recovery benefit as defined by 
Eq. (12). The mission with the highest utility is selected by a "greedy" best-first search algorithm and the planner advances to the end of this highest-utility mission. The planner then repeats this process expanding the set of all waypoints starting from the new location until an entire day of missions have been planned. Alternate search strategies may also be employed to ensure, or increase, the optimality of the result, but the best-first search is a reasonable starting point to develop a complete daily-mission plan before mission actions are required.

\section{Results}

Using the above planning and modeling infrastructure a number of planning problems were processed. All plans were developed for the Flying Fish FAA Certificate-of-Authorization (COA) test site in North Michigan where the system is deployed from the University of Michigan's biological station into the Fishtail Bay region of Douglas Lake (Fig. 7). All flight plans are constrained to the confines of Fishtail Bay. The solar model uses the clear-sky solar conditions projected for Douglas Lake on the first day of the conference to which this paper has been submitted: 29 March 2011.

The first critical test is to see if solar conditions are sufficient for overnight survival. The planner is initialized with no fixed exploration goals, a soft constraint providing $50 \mathrm{~m}$ clearance from the shore, and a hard constraint that marks the shoreline. The planner starts with a fully charged system $(\sim 3200 \mathrm{~kJ})$ and plans from one minute after midnight for two consecutive days of operation. The resulting plan (Fig. 8a) accommodates the boundary constraints with a series of flights that either maximize energy-recovery potential or minimize energy-

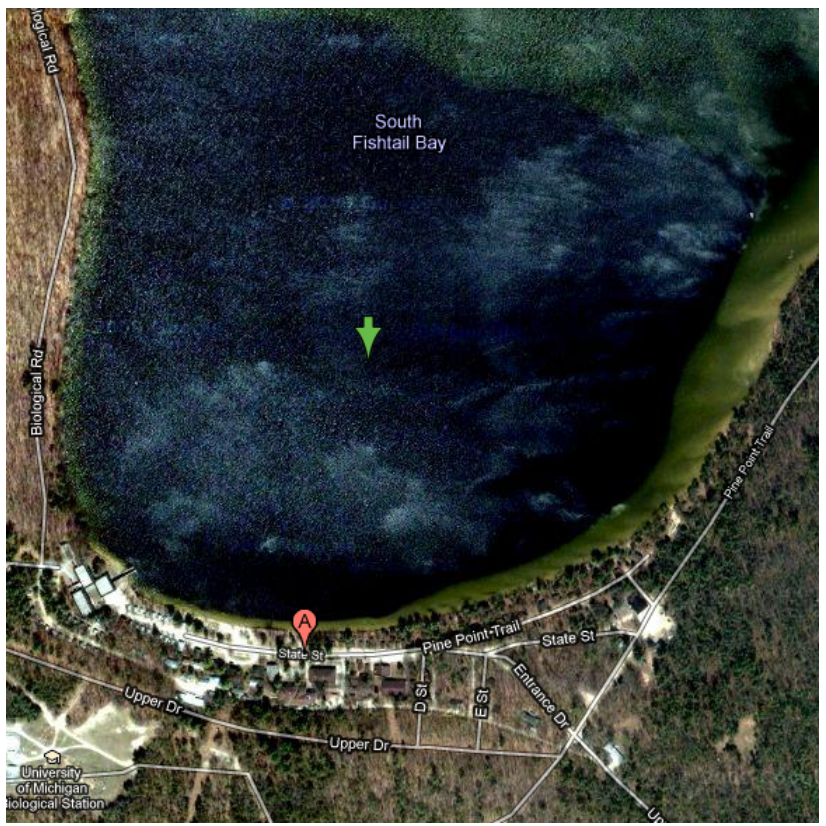

Figure 7: COA Operating Region - Douglas Lake

collection waste. The resulting balanced energy budget is shown in Fig. 8b. Notably, mission utility values

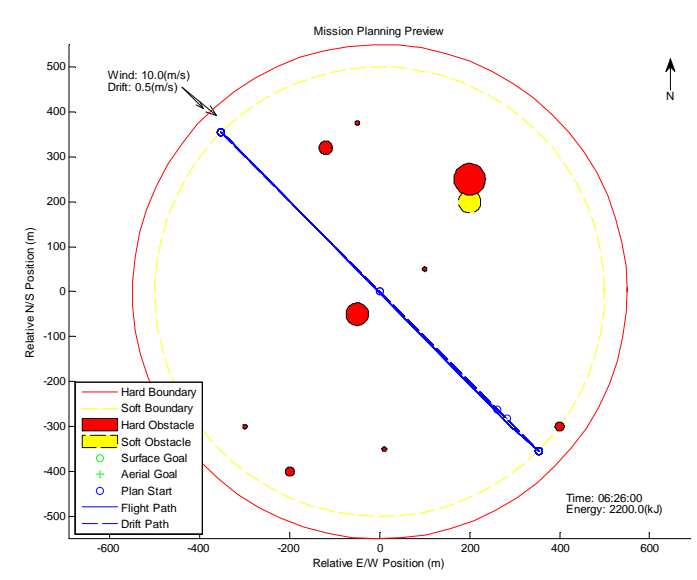

(a) Mission Plan

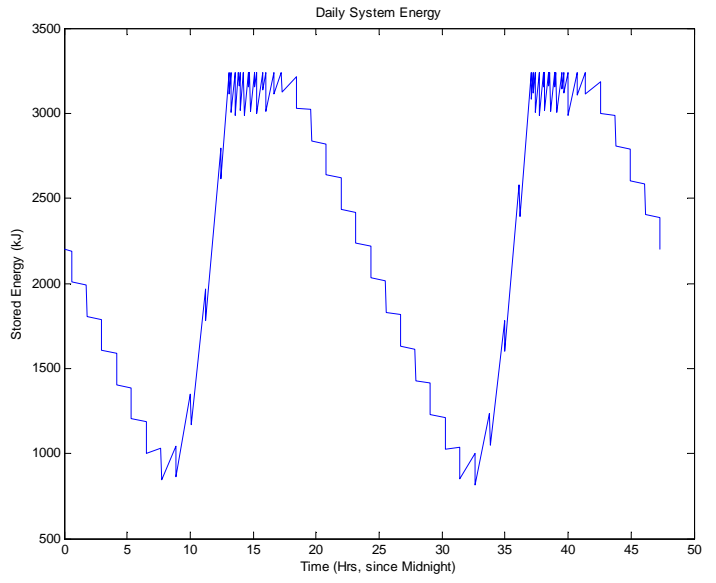

(b) Energy Budget

Figure 8: Boundary Maintenance Mission

will indicate flight operations whenever the vehicle batteries become full which is not necessarily a long term best-practice strategy. For the given location and time of year a sustainable plan can be found for moderate 
winds but persistent winds in excess of $10 \mathrm{~m} / \mathrm{s}$ can imbalance the energy budget by increasing the cost of upwind flight and rate of downwind drift. Under different solar conditions higher winds can be tolerated. For example, solar conditions at Douglas Lake in June would provide $\sim 20 \%$ more energy and a deployment closer to the equator could provide another $\sim 10 \%$ improvement with more favorable solar incidence angles.

Given that a balanced energy budget was achieved with the basic mission profile a more advanced mission was attempted. The second plan was initialized with the same conditions and constraints as the base mission with the addition of a stationary surface exploration goal. The second plan (Fig. 9a) produced another balanced daily energy budget (Fig. 9b) while visiting the exploration goal as many as 100 times in a two-day plan. Here again plan viability is subject to the accuracy of the environmental measurements. A dramatic reduction in solar insolation or increase in wind speed can tip the balance of the energy budget away from sustainability.

In order to test the system against a more challenging planning problem a set of multiple surface and airborne waypoints were combined with the base mission constraints and another two-day plan was developed (Fig. 10a). In this final case the value given to exploration in combination with the limitations of the greedy search resulted in system energy sacrifices and an imbalanced energy budget (Fig. 10b). Here the value of

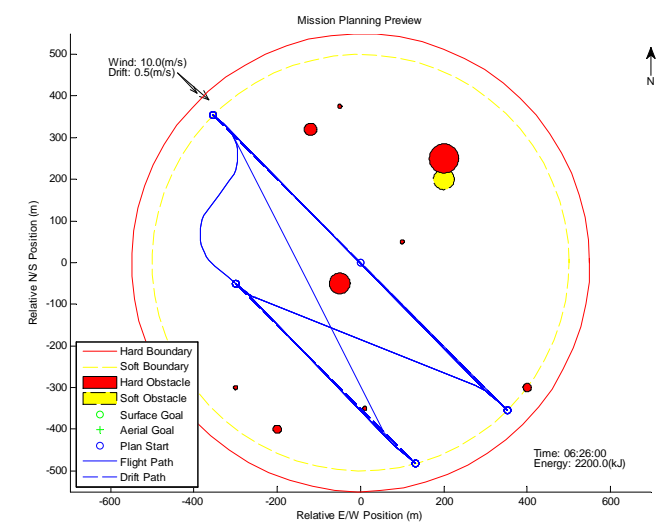

(a) Mission Plan

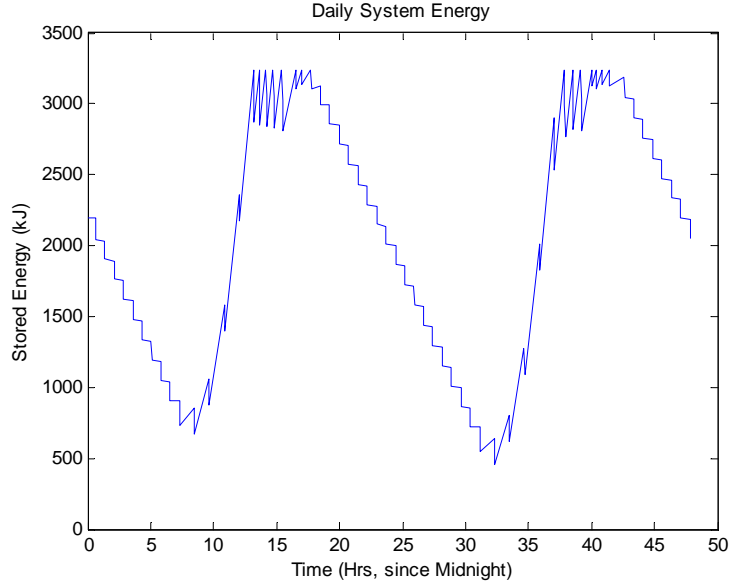

(b) Energy Budget

Figure 9: Boundary Maintenance + Surface Goal Plan

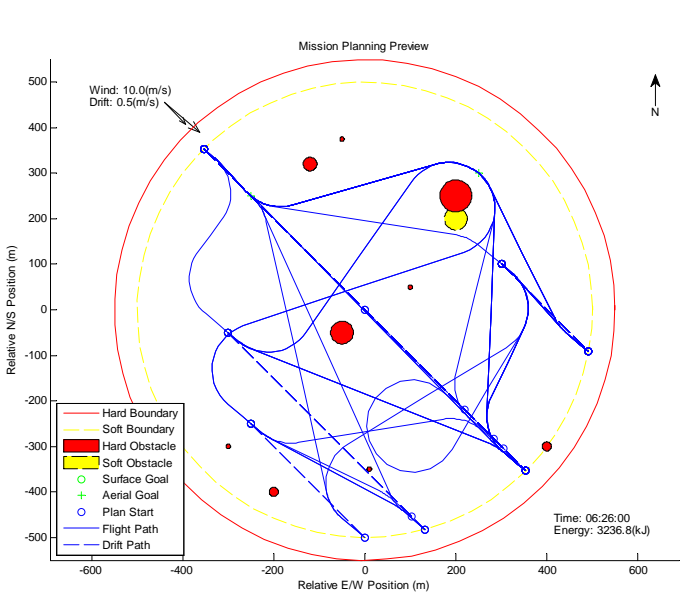

(a) Mission Plan

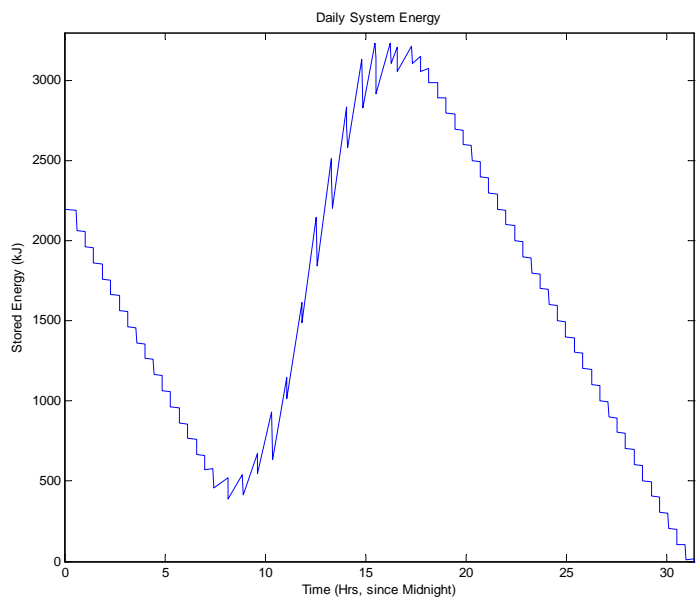

(b) Energy Budget

Figure 10: Multi-Goal Plan 
exploration becomes a critical factor as the time between visits grows for growing numbers of goal points and the increased average exploration incentive leads the planner to trade energy for goal satisfaction. Further, with increasing numbers of goal waypoints the combinatorial set of all mission paths grows exponentially and the likelihood of the best-first search finding the optimal path decreases.

\section{Conclusion}

This paper has described a comprehensive set of system and environmental models that can be used to estimate and optimize the performance of an energy-harvesting seaplane UAS. A summary of the types and variety of loads that a seaplane UAS must manage and the general challenges and benefits involved with open-ocean operation have been presented. A planning infrastructure has been described and the models and planner together have been shown capable of providing viable multi-day mission plans for a variety of pertinent seaplane UAS operations. A major goal satisfied by this work is the determination of overnight-survivable plans for the baseline constrained-operating-region mission as well as the extended basic exploration mission. The infrastructure of this planning system can now be leveraged to determine reasonable operational limits for an energy-balanced vehicle deployment.

The planner will next be applied to different operating regions, solar conditions, wind/drift speeds, and mission goals to characterize vehicle and planner limitations. There is also room in the model/planner infrastructure to increase accuracy and fidelity and to broaden the planner's capabilities. Key updates will include alternate search mechanisms for planning optimality and precise curved trajectory integrations. It might also be beneficial to explore alternate mission valuation mechanism. If an alternate valuation could be found that gives a better indication of the long-term utility of a mission the best-first search results could be improved without dramatically longer planning times. Valuations currently under consideration include mission segment duration and average rate of energy change, both of which might be used to increase the value of missions that will have higher energy harvesting potential.

\section{Acknowledgments}

The authors gratefully acknowledge the contributions of the Flying Fish team from the University of Michigan, including: Guy Meadows and Nick Wild (Naval Architecture and Marine Engineering), Brian Gilchrist, Curt Cooper, Jonathan Brown, and Gerald Giarmo (Electrical Engineering, Computer Science, and Atmospheric Oceanic \& Space Science), Luis Bernal, Pete Washabaugh, Derrick Yeo, and Stephanie Ogura (Aerospace Engineering), and Keith Shaw (BioPhysics). We also thank personnel at the University of Michigan Bio Station for supporting flight-test operations.

\section{References}

\footnotetext{
${ }^{1}$ Macy, D., Eubank, R., Atkins, E., Bernal, L., Washabaugh, P., Meadows, G., Wild, N., Smith, D., and Van Sumeren, H., "Flying Fish: A Persistent Ocean Surveillance Buoy with Autonomous Aerial Repositioning," AUVSI North America Conference, AUVSI, June 2008.

${ }^{2}$ Meadows, G., Atkins, E., Washabaugh, P., Meadows, L., Bernal, L., Gilchrist, B., Smith, D., VanSumeren, H., Macy, D., Eubank, R., Smith, B., and Brown, J., "The Flying Fish Persistent Ocean Surveillance Platform," AIAA Unmanned Unlimited Conference, Proceedings, AIAA, American Institute of Aeronautics and Astronautics, Seattle, WA, 06-09 April 2009.

${ }^{3}$ Eubank, R., Atkins, E., and Macy, D., "Autonomous Guidance and Control of the Flying Fish Ocean Surveillance Platform," AIAA Infotech@Aerospace Conference, Proceedings, AIAA, American Institute of Aeronautics and Astronautics, Seattle, WA, 06-09 April 2009.

${ }^{4}$ Liden, S., "The Evolution of Flight Management Systems," Digital Avionics Systems Conference, Proceedings, AIAA/IEEE, 1994, pp. 157-169.

${ }^{5}$ Eubank, R., Atkins, E., and Ogura, S., "Fault Detection and Fail-Safe Operation with a Multiple-Redundancy Air-Data System," Guidance, Navigation, and Controls Conference, AIAA, American Institute of Aeronautics and Astronautics, Toronto, ON, 02-06 August 2010.

${ }^{6}$ Atkins, E., Eubank, R., and Klesh, A., "A Reconfigurable Flight Management System for Small-Scale Unmanned Air Systems," AIAA Infotech@Aerospace Conference, Proceedings, AIAA, American Institute of Aeronautics and Astronautics, Seattle, WA, 06-09 Apr 2009.

${ }^{7}$ Yeo, D., Henderson, J., and Atkins, E., "An Aerodynamic Data System for Small Hovering Fixed-Wing UAS," Guidance, Navigation, and Control Conference, American Institute of Aeronautics and Astronautics, 1801 Alexander Bell Dr., Suite 500 Reston VA 20191-4344 USA,, Chicago, IL, August 2009.

${ }^{8}$ Cesnik, C., Senatore, P., Su, W., Atkins, E., Shearer, C., and Pitcher, N., "X-HALE: A Very Flexible UAV for Nonlinear
} 
Aeroelastic Tests," AIAA/ASME/ASCE/AHS/ASC Structures, Structural Dynamics, and Materials Conference, Adaptive Structures Conference, Proceedings, AIAA/ASME/ASCE/AHS/ASC, Orlando, FL, 12-15 April 2010, AIAA-2010-2715.

${ }^{9}$ Eubank, R. and Atkins, E., "Energy-Aware Flight Management and Control of the FlyingFish Solar-Powered Autonomous Seaplane," Unmanned Systems North America, AUVSI, AUVSI, Denver, CO, 24-27 August 2010.

${ }^{10}$ Klesh, A., Optimal Exploration Systems, Ph.D. thesis, Univ. of Michigan, Dept. of Aerospace Engineering, 2009.

${ }^{11}$ Dubins, L., "On curves of Minimal Length with a Constraint on Average Curvature, and with Prescribed Initial and Terminal Positions and Tangents," American Journal of Mathematics, Vol. 79, No. 3, 1957, pp. 497-516.

12 Johnson, V., Pesaran, A., and Sack, T., Temperature-Dependent Battery Models for High-Power Lithium-Ion Batteries, National Renewable Energy Laboratory, 2001.

${ }^{13}$ Chen, M. and Rincon-Mora, G., "Accurate Electrical Battery Model Capable of Predicting Runtime and IV Performance," Energy Conversion, IEEE Transactions on, Vol. 21, No. 2, 2006, pp. 504-511.

${ }^{14}$ Gao, L., Liu, S., and Dougal, R., "Dynamic lithium-ion Battery Model for System Simulation," IEEE transactions on components and packaging technologies, Vol. 25, No. 3, 2002, pp. 495-505.

${ }^{15}$ Kroeze, R. and Krein, P., "Electrical Battery Model for Use in Dynamic Electric Vehicle Simulations," Power Electronics Specialists Conference, 2008. PESC 2008. IEEE, IEEE, 2008, pp. 1336-1342.

${ }^{16}$ Michalsky, J., "The Astronomical Almanac's Algorithm for Approximate Solar Position (1950-2050)," Solar Energy, Vol. 40, No. 3, 1988, pp. 227-235.

${ }^{17}$ Reda, I. and Andreas, A., "Solar Position Algorithm for Solar Radiation Applications," Tech. Rep. 5, National Renewable Energy Laboratory, Golden, CO, January 2008, NREL/TP-560-34302. 